\title{
Advanced septa size quantitation determines the evaluation of histological fibrosis outcome in chronic hepatitis B patients
}

\author{
Bingqiong Wang ${ }^{1} \cdot$ Yameng Sun ${ }^{1} \cdot$ Jialing $\mathrm{Zhou}^{1} \cdot$ Xiaoning $\mathrm{Wu}^{1} \cdot$ Shuyan $\mathrm{Chen}^{1} \cdot$ Shanshan $\mathrm{Wu}^{1} \cdot \mathrm{Hui}^{\mathrm{Liu}}{ }^{2} \cdot$ \\ Tailing Wang ${ }^{3} \cdot$ Xiaojuan Ou ${ }^{1} \cdot$ Jidong $\mathrm{Jia}^{1} \cdot$ Hong You ${ }^{1}$
}

Received: 4 January 2018 / Revised: 8 March 2018 / Accepted: 9 March 2018 / Published online: 21 May 2018

(c) The Author(s) 2018. This article is published with open access

\begin{abstract}
Hepatitis B (HBV)-related fibrosis can be reversed after effective antiviral therapy. However, detailed changes of collagen characteristics during fibrosis regression remain unclear. Paired biopsy samples obtained from chronic hepatitis B patients were imaged with second harmonic generation/two photon excitation fluorescence (SHG/TPEF)-based microscopy to identify and quantify collagen features in portal, septal, and fibrillar areas. According to the changes of Ishak stage and qFibrosis score, a total of 117 patients with paired liver biopsy appeared to have four different outcomes after 78-week antiviral therapy: fast reverse (9\%), reverse $(63 \%)$, stable $(15 \%)$, or progress $(13 \%)$ on fibrosis. Among 71 collagen features identified by SHG/TPEF analysis, the most prominent fibrosis reversion occurred in the "septal" area, followed by the "fibrillar" area, but not in the "portal" area $(P<0.001)$. Further analysis of 1060 individual septa identified four parameters that correlated with fibrosis reversion: average width, maximum width, number of fibers, and number of cross-link fibers $(P$ $<0.001$ ). Average septal width was independently associated with regressive septa (odds ratio $(\mathrm{OR})=5.22,95 \%$ confidence interval (CI): 4.17-6.53; $P<0.001$ ), with an AUROC of 0.96 (95\% CI: 0.95-0.97). The threshold used to discriminate reversal of fibrosis was $30 \mu \mathrm{m}$. In conclusion, septal collagen was determined to be the most useful histological feature for evaluation of dynamic changes in liver fibrosis. Septal width was the most predictive indicator of prognosis in liver fibrosis.
\end{abstract}

\section{Introduction}

Liver fibrosis is a dynamic process resulting from the wound-healing response to liver injury of any etiologies [1]. Chronic hepatitis B is a major cause of liver fibrosis and cirrhosis. Liver fibrosis and early cirrhosis can be reversed

Electronic supplementary material The online version of this article (https://doi.org/10.1038/s41379-018-0059-x) contains supplementary material, which is available to authorized users.

Hong You

youhong30@sina.com

1 Liver Research Center, Beijing Key Laboratory of Translational Medicine in Liver Cirrhosis, National Clinical Research Center for Digestive Disease, Beijing Friendship Hospital, Capital Medical University, Beijing, China

2 Department Pathology, Beijing Youan Hospital, Capital Medical University, Beijing, China

3 Department Pathology, China-Japan Friendship Hospital, Beijing, China after effective antiviral therapy in chronic hepatitis B patients has become increasingly evident [2-6].

Liver biopsy has traditionally been regarded as the gold standard for evaluation of fibrosis reversion. It was defined as at least one-point decrease after antiviral therapy based on semi-quantitative scoring systems in most clinical trials $[4,6]$. These semi-quantitative scores could partly reflect the architectural distortion. However, the changes of detailed collagen structural characteristics have not been identified. There is a need for further refinements in the histological assessment of liver fibrosis reversion.

Liver fibrosis is characterized by the deposition of excessive extracellular matrix enriched in cross-linked collagen type I/III [7, 8]. During liver fibrosis progression in chronic viral heptatitis, collagen deposition extends from portal tracts to adjacent parenchyma accompanied by the formation of portal/periportal fibrosis, septal fibrosis, and perisinusoidal fibrosis $[9,10]$. But changes of collagen pattern during the regression of liver fibrosis was still unknown. By a few studies focused on the histopathologic pattern of regressing fibrosis, based on a sequential biopies, a landmark study from Ian Wanless and colleagues found 
thinning of fibrous septa could represent the important sign of fibrosis reversion [11]. However, this point of view mainly based on liver transplant biopies from crosssectional study. Dynamic changes of collagen features and collagen patterns during fibrosis regression pre- and posttreatment still remain unidentified.

Recently, second harmonic generation/two photon excitation fluorescence (SHG/TPEF)-based microscopy technology has been widely used in liver fibrosis [12-16]. It has been demonstrated to have a strong association with fibrosis stage by comprehensive qualitative and quantitative measurements of collagen content [13, 14]. Besides, it has been shown to monitor the structural progression of collagen at all stages of liver fibrosis [15]. Most importantly, it can provide both the morphological characteristics and spatial distributions of the collagen [17]. In this study, we aim to use this fully automated approach to identify the changes of collagen features during the liver fibrosis reversion in chronic hepatitis B patients pre- and post- treatment.

\section{Materials and methods}

\section{Patients}

Liver biopsies were performed in chronic hepatitis B patients before and after 78 weeks of therapy. The enrollment of the study cohort has been described previously [18]. In brief, treatment-naive patients were eligible for recruitment if they aged at 18-65 years, had been tested positive for hepatitis B surface antigen (HBsAg) $>6$ months, positive for $\mathrm{HBeAg}$ with Hepatitis $B$ virus (HBV)DNA levels higher than $20,000 \mathrm{IU} / \mathrm{mL}$ or negative for HBeAg with HBV-DNA levels higher than $2000 \mathrm{IU} / \mathrm{mL}$, had a liver biopsy showing an Ishak fibrosis score $\geq 3$ at baseline or week 78 after treatment with qualified biopsy.

During the study, clinical, laboratory, and imaging assessments were made at baseline and every 3 months thereafter during follow ups. Assessments of HBV-DNA by sensitive real-time PCR assay and HBV serological markers were performed at baseline and subsequently every 24 weeks. Liver biopsy was performed at baseline and week 78 after treatment.

The study was conducted in compliance with the ethical principles of the Declaration of Helsinki. The study protocol was approved by the ethics committee at each participating facility. Patients gave written informed consent for participation in the study (NCT01938781, NCT01938820).

\section{Histological assessment}

Liver biopsies were fixed in $10 \%$ buffered formal saline and embedded in paraffin wax for subsequent histological analysis. Tissue sections, $5 \mu \mathrm{m}$ thick, were stained with hematoxylin and eosin, Masson's Trichrome, and Reticulin for standard histological assessment. The degree of inflammation was graded by using the Ishak modified histology activity index (HAI) grading system and fibrosis was staged by Ishak fibrosis scoring system [9] by two senior experts (HL and TLW). Besides, all of the enrolled liver biopsy was further evaluated by Beijing classification (HL and YMS). Histological evaluations were performed blindly [18].

\section{SHG/TPEF imaging and processing}

All unstained liver biopsy specimens were imaged by a SHG/ TPEF technology (Genesis200 ${ }^{\mathrm{TM}}$, HistoIndex Pte. Ltd, Singapore). A magnified image (20X) of a specimen was captured for further image-analyzing. Each image was saved as a resolution of $512 \times 512$ pixels. Needle biopsies were entirely imaged and stitched by multiple images. A total of 100 features showing numbers, length, width, and cross-link of collagen fibers from portal, septal, and fibrillar areas in the raw SHG images were extracted to reflect the collagen structural information, detailed information as described previously in the study of $\mathrm{Xu}$ et al. [17]. Correlation analysis and Bayesian Information Criterion test was used to select the most related features to establish qFibrosis. At last, a total of 15 features was selected as the most important features: (1) Total: collagen percentage area, string length, string width, string solidity, string perimeter, (2) Portal: aggregated and distributed collagen percentage area, number of strings and cross-link collagen fibers (3) Septal: number of thick strings and cross-link collagen fibers, string length (4) Fibrillar: collagen percentage area, number of strings and cross-link collagen fibers. By using the nonlinear regression model, we combine these selected collagen features into a single index according to the distribution of fibrosis stages. The qFibrosis was calculated by the following equation:

$q$ Fibrosis $=\varepsilon+\sum_{i=1}^{N}\left(a_{i} X_{i}+b_{i} \log \left(X_{i}\right)\right)$

Matlab 2015a was used to select features and model fitting (The MathWorks, Inc., Natick, MA).

The change of each parameter in different regions was defined by using the normalized $\log 2$ (parameter value in week 78/parameter value at baseline). The normalized mean scores were visualized in a heatmap to compare the changes of collagen features across different fibrosis regression group and different regions before and after therapy.

\section{Quantitation of septal area}

The raw SHG/TPEF images of liver biopsy specimens were analyzed using digital imaging analysis by an automated 
segmentation algorithm to detect and quantify the septal area. Using histological diagnosis of septa as gold standard, the final analysis was conducted after the automated identification reaching an excellent agreement with histological diagnosis. Then quantify the whole characteristics of septal area, the numbers and proportions of fibers and cellular regions inside the region of septa. Beijing classification was used to define the progressive septa and regressive septa [18].

\section{Statistical analyses}

Data are presented as the proportion, mean $\pm \mathrm{SD}$, and median with the interquartile range. One-way analysis of variance and Kruskal-Wallis test were used to assess the differences for continuous variables between groups. $\chi^{2}$ tests were undertaken for categorical variables between groups. Spearman rank correlation coefficient was used for correlations between variables. Univariate and multivariate analysis was used to identify parameters related with regressive septa. Diagnostic value and cutoff values were identified by operating characteristic curve (ROC). Twosided $P$ values $<0.05$ were considered significant. Data were analyzed using SPSS 22.0.

\section{Results}

\section{Clinical characteristics of patients}

A total of 146 patients who have paired liver biopsies with significant fibrosis (Ishak stage $\geq 3$ ) were enrolled. Twentytwo patients with unqualified paired biopsies (the length of liver biopsy $<1 \mathrm{~cm}$ ) and another seven patients of inadequate quality to be informative for morphometric image analysis were excluded. Finally, 117 patients were included in the study. The flow chart of our study is shown in Fig. 1.

Liver biopsy samples were evaluated to determine changes in Ishak stage and qFibrosis score. The response of fibrosis to 78-week antiviral therapy was categorized as: fast reverse (decrease in Ishak stage $\geq 2 ; 9 \%$ ), reverse (decrease in Ishak stage $\geq 1$ or decrease in qFibrosis score $\geq 0.5 ; 63 \%$ ), stable (increase or decrease in qFibrosis score $\leq 0.5 ; 15 \%$ ), or progress (increase in Ishak stage $\geq 1$ or increase in $\mathrm{qFi}$ brosis score $\geq 0.5 ; 13 \%$ ). Because reversal rate was found to differ significantly among groups, this classification scheme was used to categorize histological features related to fibrosis reversion.

Demographic, clinical, and histological characteristics are detailed in Supplementary Table 1. Patients were predominantly male $(81.2 \%)$, with a median age of 38 years old. There were no statistical differences of demographic and clinical features between the four groups at baseline.

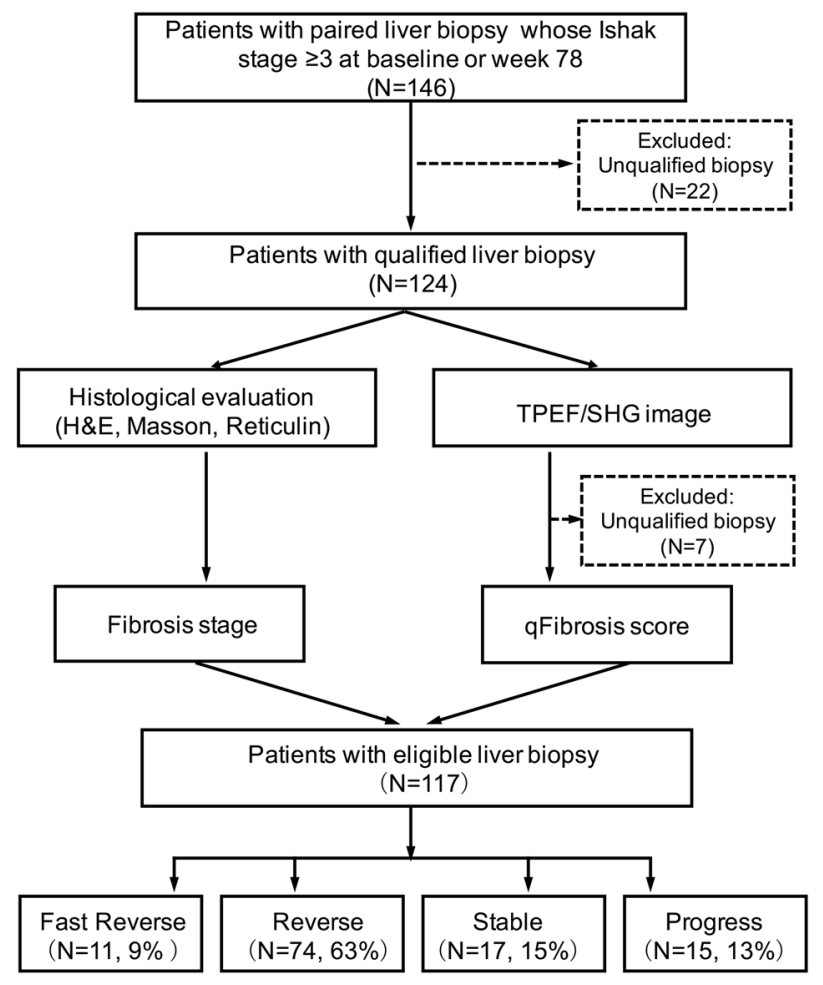

Fig. 1 Flow chart of the selection process and grouping of this study population

After 78-week therapy, significant decreases were observed in alanine aminotransferase (from $80.5 \mathrm{U} / \mathrm{L}$ to $28.0 \mathrm{U} / \mathrm{L}$, $P<0.001$ ), aspartate aminotransferase (from $52.0 \mathrm{U} / \mathrm{L}$ to $25.0 \mathrm{U} / \mathrm{L}, P<0.001)$, aspartate aminotransferase to platelet ratio index (APRI) score (from 0.8 to $0.4, P<0.001$ ), fibrosis-4 (FIB-4) index (from 1.5 to $1.1, P<0.001$ ), and liver stiffness (from $12.3 \mathrm{kPa}$ to $6.9 \mathrm{kPa}, P<0.001$ ). Decreases in albumin, APRI score, FIB-4 score and LSM value were more readily apparent in the fast reverse and reverse groups, as compared with the stable and progress groups $(P<0.001)$.

\section{Histological assessment of liver biopsy}

The dynamic changes of histological features between the four groups are shown in Table 1. Interestingly, the proportion of advanced fibrosis was higher in fast reverse and reverse groups than the other two groups $(72.8 \%, 66.2 \%$, $23.5 \%$, and $40.0 \%$, respectively, $P=0.002$ ). The severity of necroinflammation was similar in each group at baseline $(P=0.107)$. After 78 weeks of treatment, the median Ishak stage decreased from 4 to 3 and qFibrosis score decreased from 3.6 to $2.7(P<0.001)$. The median Ishak histology activity index score decreased significantly (from 7 to 4 , $P=0.001)$. Representative cases with paired liver biopsies in each group are shown in Fig. 2. Either in the fast reverse or reverse group, most of the thick, inflamed, edematous 
Table 1 Dynamic change in histological features between the four group

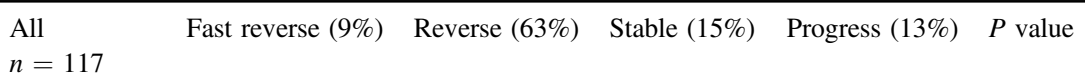

$\begin{array}{lll}4(5.4) & 1(5.9) & 2(13.3) \\ 25(33.8) & 8(47.1) & 8(53.3) \\ 24(32.4) & 8(47.1) & 3(20.0) \\ 21(28.4) & 0(0.0) & 2(13.3)\end{array}$

0.107

$0.002 * *$

$\begin{array}{llll}0(0.0) & 0(0.0) & 2(13.3) & \\ 25(33.8) & 13(76.5) & 7(46.7) & \\ 32(43.2) & 3(17.6) & 5(33.3) & \\ 16(21.6) & 0(0.0) & 1(6.7) & \\ 1(1.4) & 1(5.9) & 0(0.0) & \\ 3.7(2.9,4.7) & 3.2(2.5,3.6) & 2.3(1.9,3.8) & 0.001^{* * *} \text { * } \\ & & & 0.654\end{array}$

$\begin{array}{lll}29(39.2) & 7(41.2) & 3(20.0) \\ 43(58.1) & 10(58.8) & 11(73.3) \\ 2(2.7) & 0(0.0) & 1(6.7) \\ 0(0.0) & 0(0.0) & 0(0.0)\end{array}$

Data presented as median (lower quartile, upper quartile) or number (\%)

HAI Histology activity index

septa changed to acellular, thin or delicate inactive septa. But it did not have the same tendency of changes in the other two groups.

\section{Specific change of collagen architectural pattern in SHG/TPEF analysis}

Further, the change of multiple morphological collagen architectural features identified by SHG/TPEF was used to refine the whole collagen architectural change. Among 100 features quantified by SHG/TPEF-based technology, only 71 informative parameters were included in the final analysis, 29 parameters with the number "0" (nothing detected) were excluded.

The normalized mean value in each group was visualized in a heatmap (Fig. 3a). The majority of quantified parameters of the whole biopsy had a gradient decreasing trend from stable group to fast reverse group according to 


\section{A Fast Reverse}

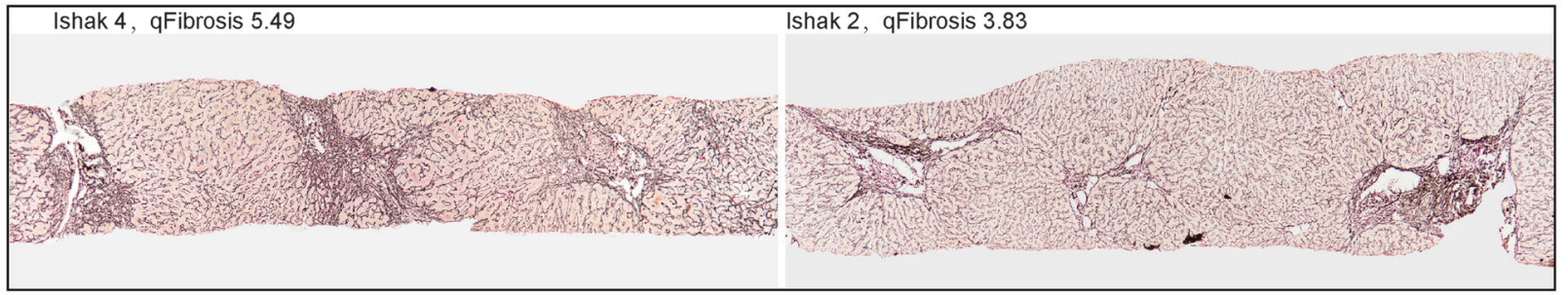

B Reverse

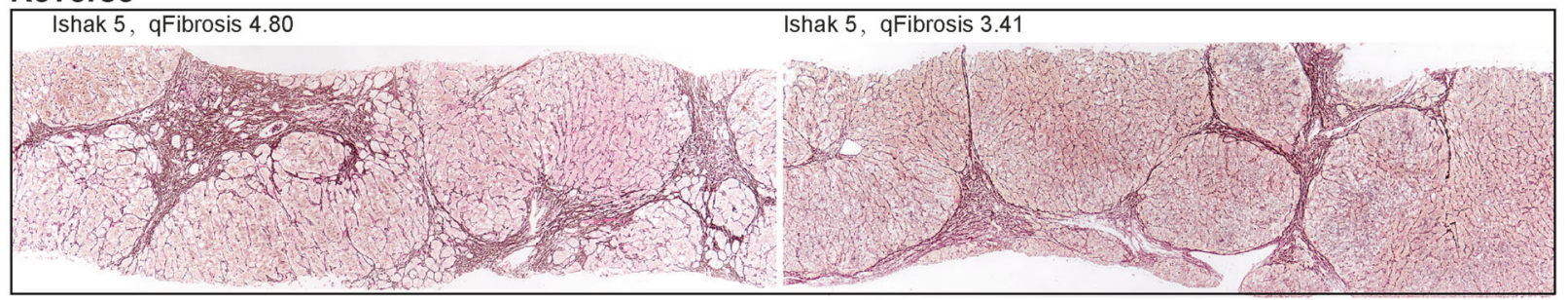

C Stable

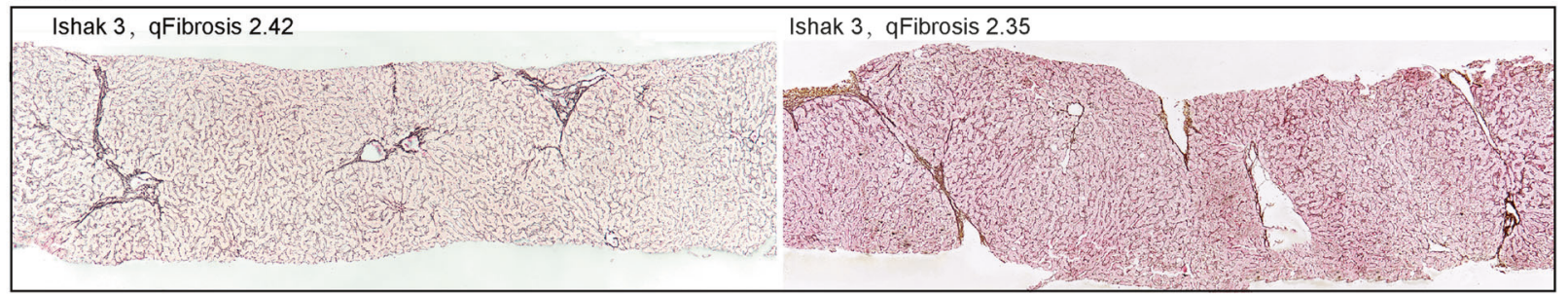

D Progress

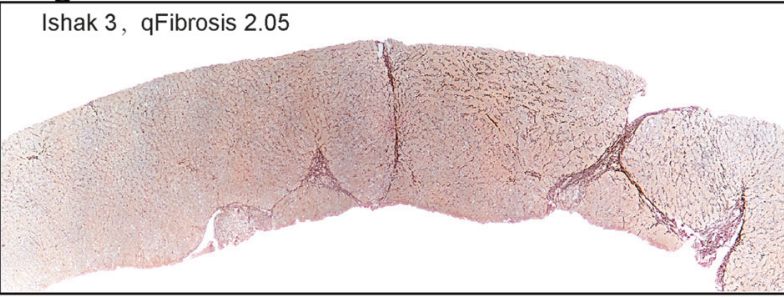

Fig. 2 Representative reticulin stained liver biopsy samples of chronic hepatitis B patients in different group. a The liver biopsy samples of chronic hepatitis B patients in fast reverse group, whose fibrosis stage decreased from Ishak 6 to Ishak 2, qFibrosis score decreased from 5.49 to 3.83. $\mathbf{b}$ The liver biopsy samples of chronic hepatitis B patients in reverse group, whose qFibrosis changed from 4.80 to 3.41 before and

changes of collagen features, whereas increased in progress group. In the three parts of collagen pattern, the most prominent fibrosis changes occurred in septal area, which was consistent with the change of total collagen pattern. The correlations of these collagen architectural parameters related with different group were expressed by spearman correlation coefficient (Supplementary Table 2). The correlation was from 0.32 to 0.68 in parameters of septa, from 0.30 to 0.68 in parameters of fibrillar, from -0.04 to 0.15 in parameters of portal. Most of the selected parameters in "septal" area change significantly across the four groups, followed by "fibrillar" area, "portal" area ( $80 \%$ vs. $20 \%$ vs. $0 \%$, respectively, $P<0.001)$. So, the histological changes
Ishak 3, qFibrosis 2.74

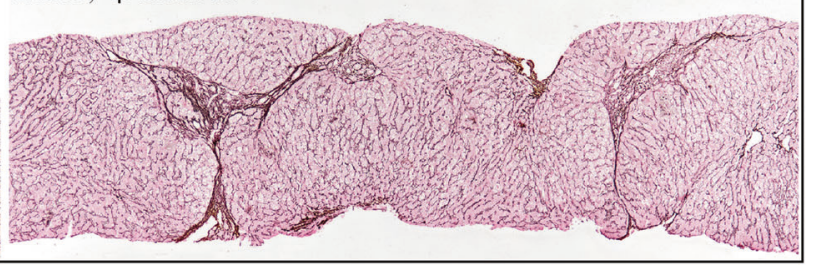

after treatment, although Ishak score remained in stage 5. c The liver biopsy samples of chronic hepatitis B patients in stable group, whose qFibrosis score change within 0.5. d The liver biopsy samples of chronic hepatitis B patients in progress group, whose qFibrosis changed from 2.05 to 2.74 , although Ishak score remained in stage 3

happened in septal area were useful to refine the histological characteristics during fibrosis reversion.

Figure $3 \mathrm{~b}$ shows the dynamic change of collagen proportionate area quantified by SHG/TPEF-based technology from different collagen structures. A meaningful increasing trend of total collagen proportionate area, septal collagen proportionate area, fibrillar collagen proportionate area was found in the four groups $(-60.68 \%$ vs. $-43.25 \%$ vs. $+3.68 \%$ vs. $+59.65 \%$ and $-57.2 \%$ vs. $-43.0 \%$ vs. $+15.4 \%$ vs. $+63.6 \%$ and $-61.7 \%$ vs. $-48.1 \%$ vs. $-2.8 \%$ vs. $+46.3 \%$ in fast reverse group, reverse group, stable group and progress group, respectively). The difference has statistical significance between fast reverse/reverse group 

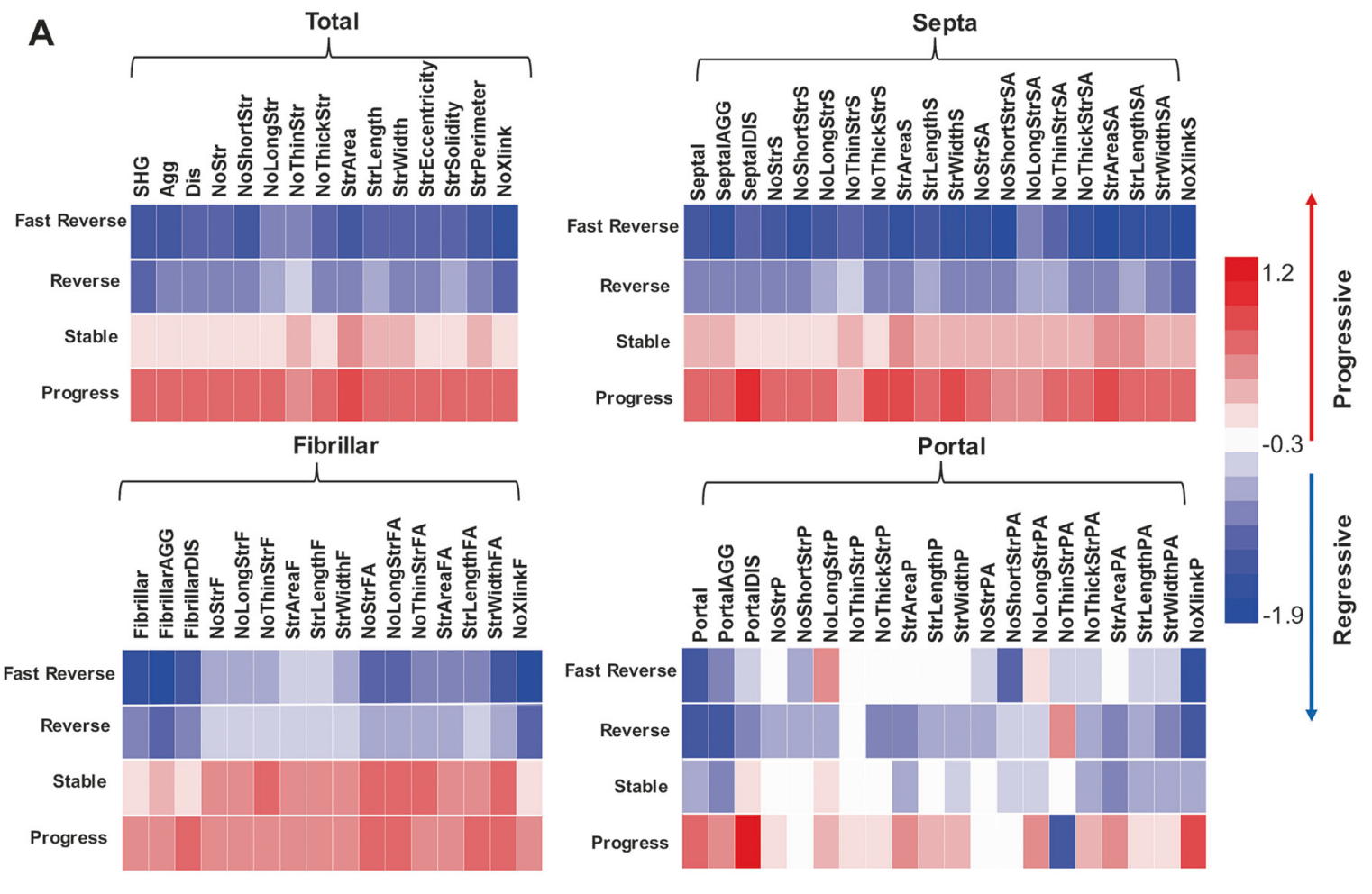

B
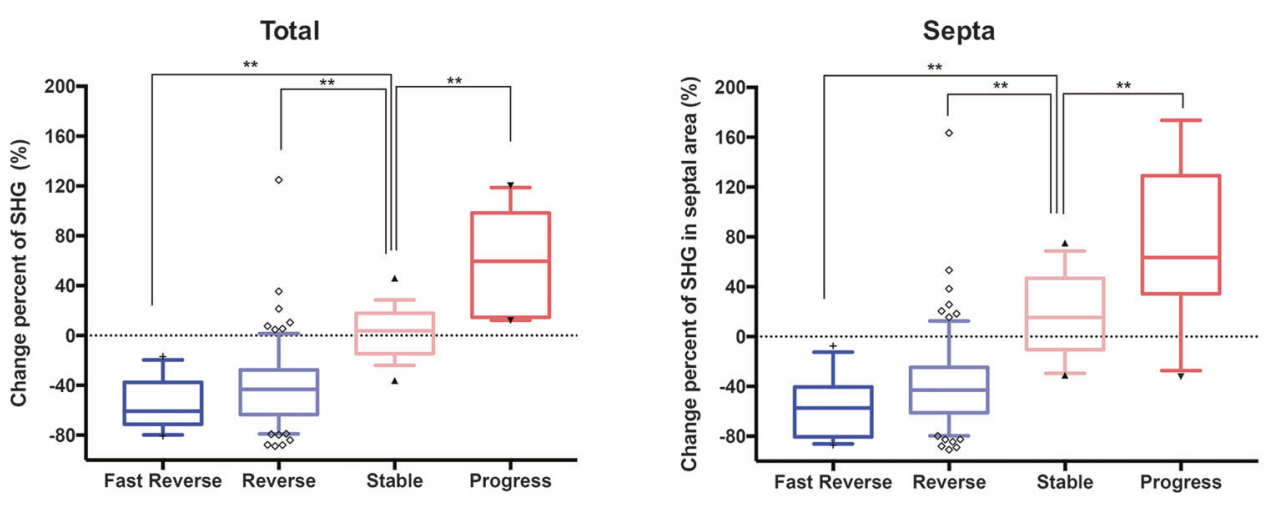

Fibrillar

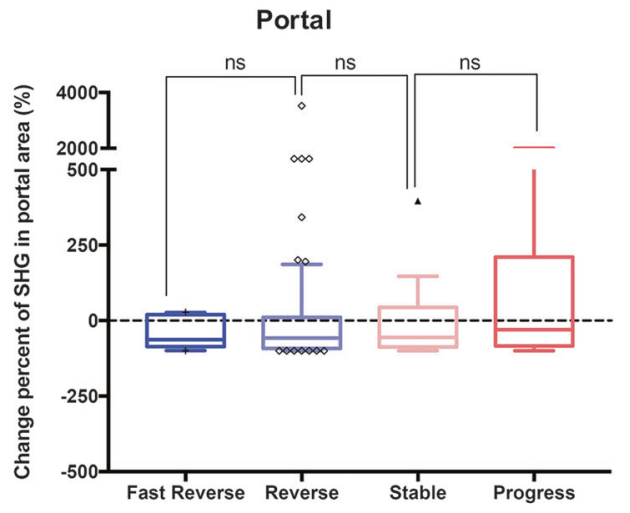

Fig. 3 Dynamic changes in different collagen structure features quantified by SHG/TPEF-based technology pre- and post treatment. a Heatmap of change of multi-morphological collagen features in total, septal, fibrillar, and portal regions pre- and post treatment. Parameters in general, portal, septal, and perisinusoidal regions were normalized by Lg2 (Mean_78w/Mean_0w). b The change of collagen proportionate area quantified by SHG/TPEF based image analysis of different

collagen pattern before and after treatment in different groups. The percentage change was defined as (Value_78w-Value_Baseline)/ Value_Baseline, $* P<0.05 ; * * P<0.001$ (Mann-Whitney $U$-test). SHG: Collagen percentage area quantified by SHG/TPEF. Agg: Aggregated collagen; Dis: Distributed collagen; NoStr: Number of Collagen Strings; NoXlink: Number of Cross-link Strings 


\section{A. SHG/ TPEF image}

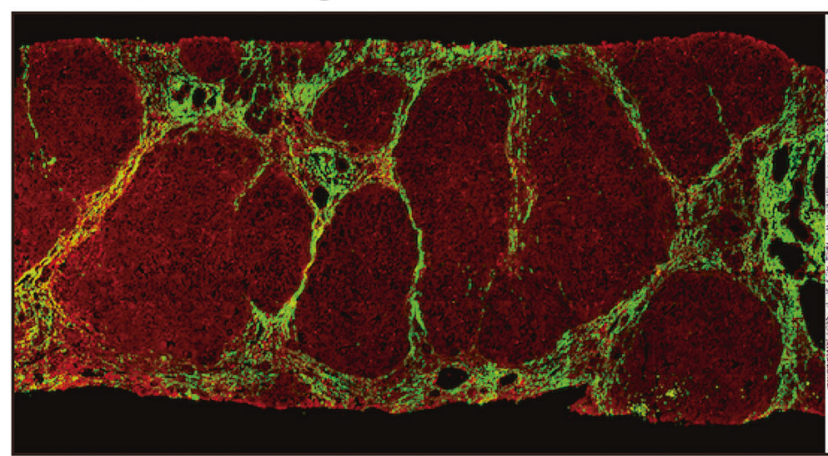

\section{Digital image analysis of septal area}

(a) Detection of septal area

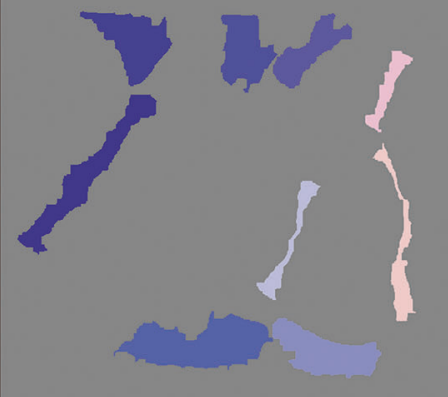

(b) Fibers within septal area

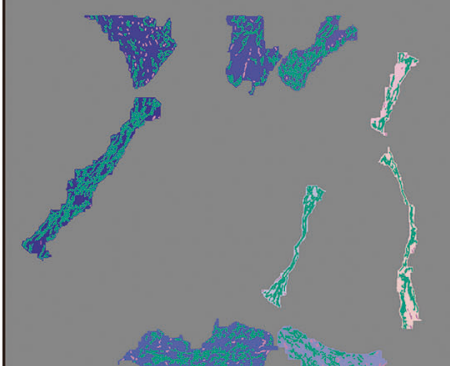

(c) Celluar region within septal area
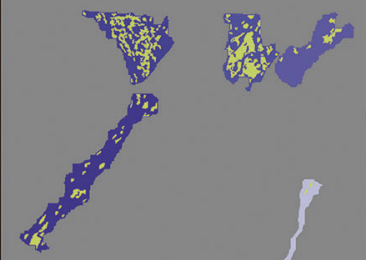

as
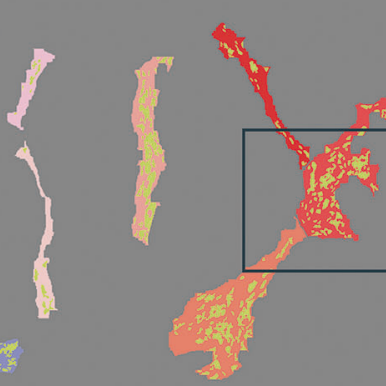

Fig. 4 The process of automated image analysis. One fragment in a liver biopsy specimen of Ishak 6 stage is shown for example. a, b SHG/TPEF microscopy image and reticulin stained image. c Autodetection of septal area and quantification of septa. Each color represented a septal area, the color inside the septal area represented fibers and different cell region. The quantification of septa includes the

\section{B. Reticulin stain}
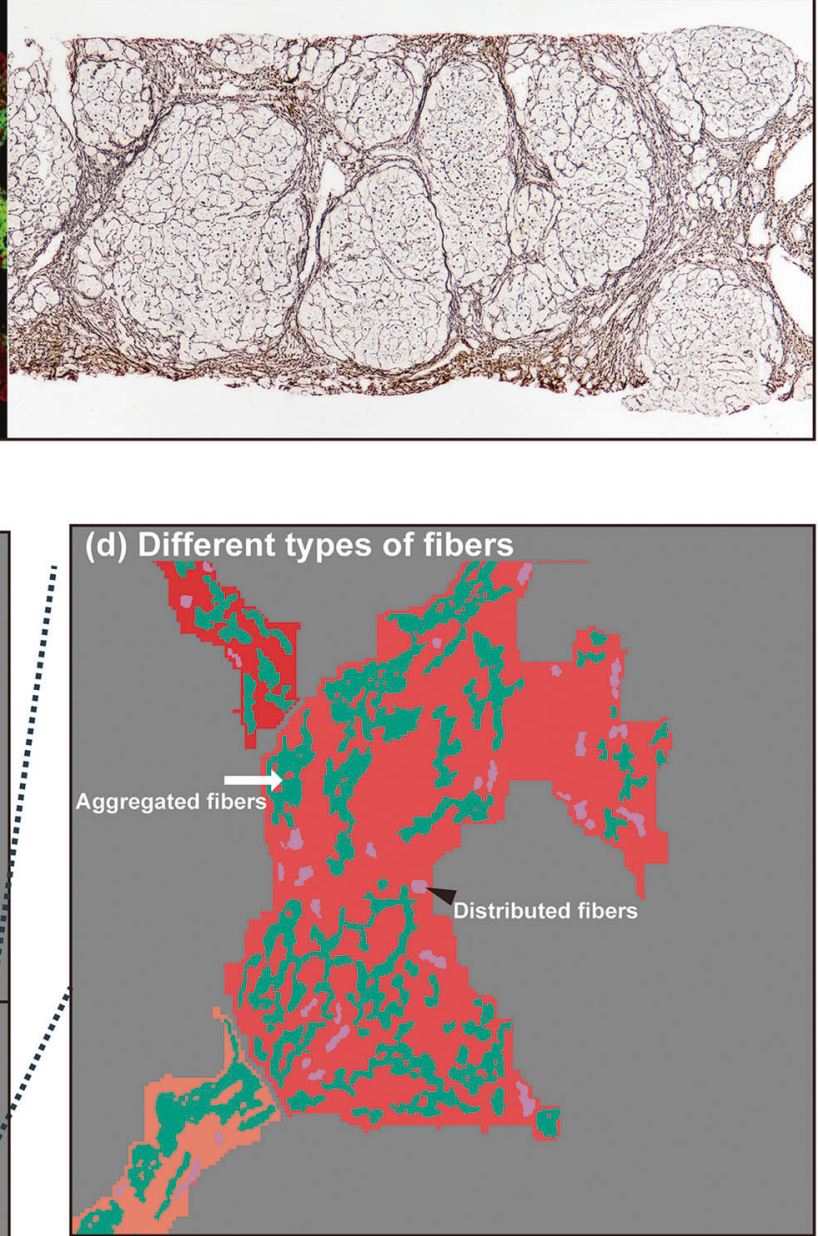

(e) Cellular regions

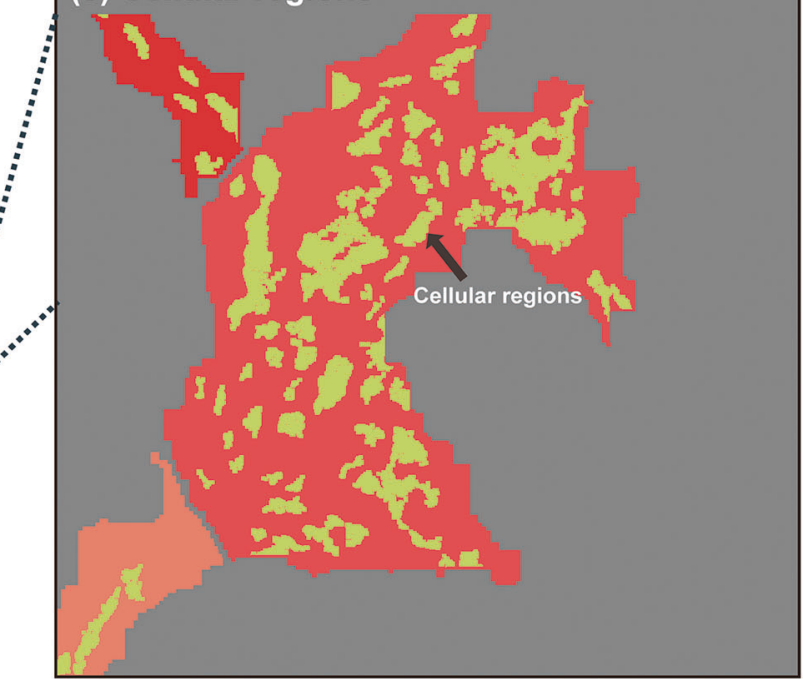

whole characteristics of septa region (a), the number and proportions of fibers (b) and cellular regions inside the region of septa (c). Right panel shows magnification of selected septal areas (d, e). In the upper right image, the white arrow indicates the aggregated fibers; black arrowheads indicate the distributed fibers. In the bottom right image, black arrows indicate cellular region 

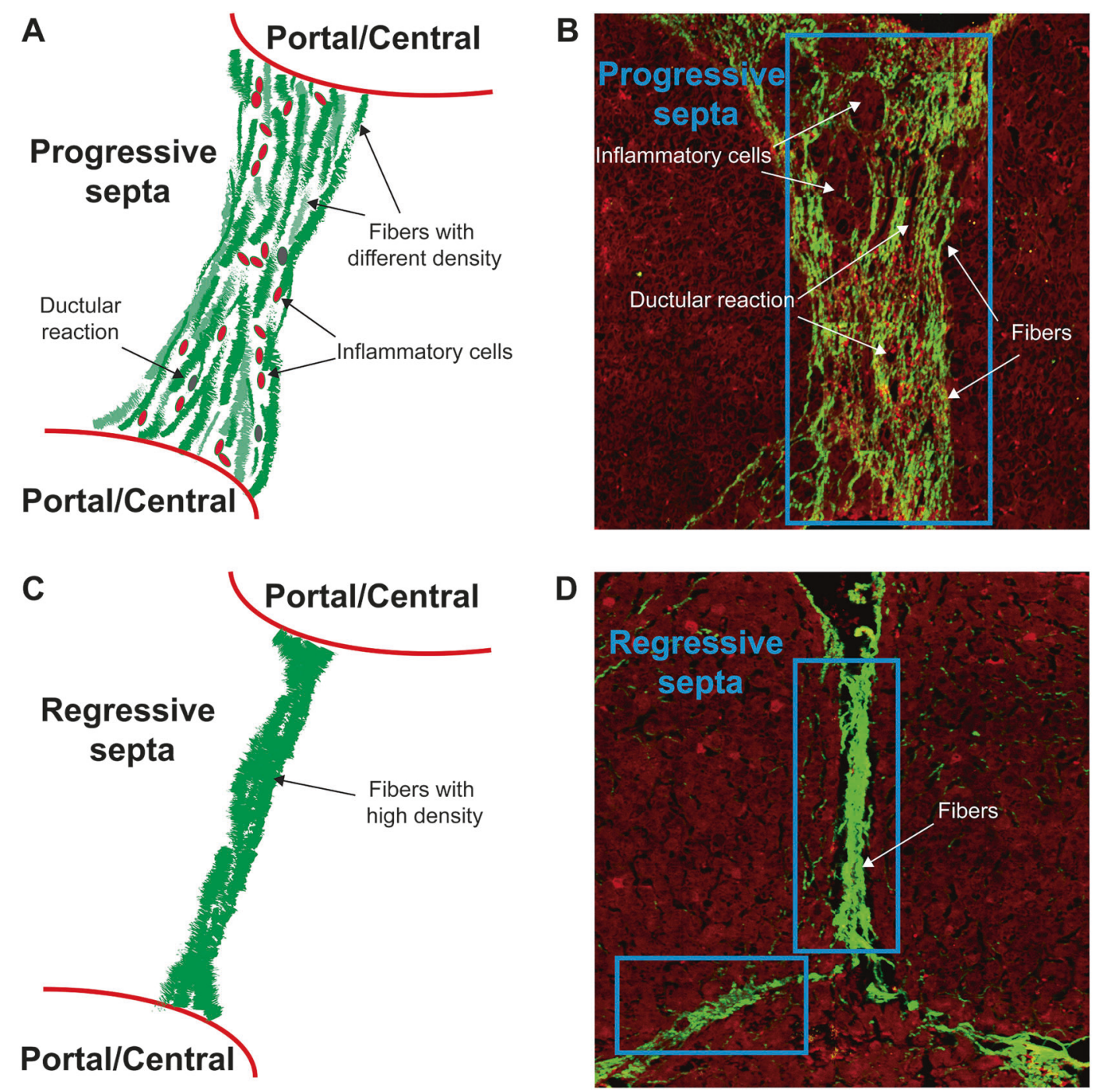

Fig. 5 Schema of two kinds of septa and imaging analysis process of septal area in chronic hepatitis B patients with significant fibrosis. a, b Schema and imaging analysis process of progressive septa. c, $\mathbf{d}$ Schema and imaging analysis of regressive septa

and stable/progress group (all $P<0.001$ ). The difference was more prominent in "septal" area than the other two pattern, followed by the changes occurred in "fibrillar" area.

\section{Refined quantitative characteristics of septal area}

The quantitative characteristics of septal area were evaluated (Fig. 4a-c). The representative SHG/TPEF images and corresponding reticulin stain images are shown in Fig. 4a, b. Figure $4 c$ (a)-(e) showed the detection and quantification of the different types of fibers and cellular regions inside the septal region. According to Beijing classification, two kinds of septal fibrosis were defined (Fig. 5a-d). There were 497 regressive septa and 582 progressive septa in 117 paired liver biopsy.

Next, to find the differences between the two kinds of septa, a total of 30 new parameters from the quantitative parameters of septal area including 10 characteristics of whole septa, 16 characteristics of fibers and four cellular regions inside the region of septa were used to refine the septal area (Supplementary Table 3). Among these characteristics, a total of four parameters including average width, maximum width, number of fibers and number of cross-link fibers, were strongly correlated with the type of septa $\left(P<0.001\right.$ for all, $\left.\gamma_{\mathrm{s}}=0.71-0.78\right)$ according univariate analysis (Table 2 ).

In multivariable analysis, the average width $(\mathrm{OR}=5.22$, 95\% confidence interval $(\mathrm{CI}): 4.17-6.53 ; P<0.001)$ of septal area was independently correlated with the reversibility of septal fibrosis. For a 10- $\mu \mathrm{m}$ change in average septal width, the probability of progressive septa increased 5.22-fold. For discrimination between regressive septa vs. progressive septa, the area under the curve was 0.96 (95\% CI: 0.95-0.97, $P<0.001$, Supplementary Figure 1). 


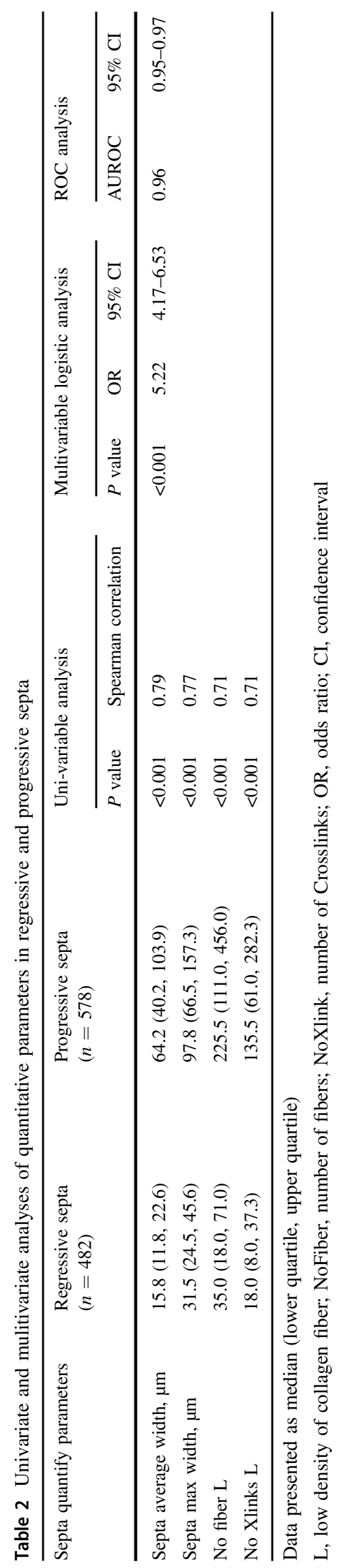

According to ROC curve analysis, the best compromise between sensitivity $(86.9 \%, 95 \% \mathrm{CI}: 83.8-89.5 \%)$ and specificity $(91.5 \%, 95 \%$ CI: $88.6-93.8 \%)$ was found for a threshold of $30 \mu \mathrm{m}$.

\section{Discussion}

Precise assessment of liver fibrosis reverse is meaningful to estimate the efficacy of treatment and predict clinical outcome. The present study systematically investigated the detailed changes of collagen characteristics during regression of liver fibrosis by a fully automated SHG/TPEF based quantitative method.

Septal area was demonstrated as the most prominent histological feature in the dynamic change of fibrosis by comparing the histological change in four groups with different reversal rate to simulate a fibrosis regression model. The importance of septal area in predicting the reversibility of liver fibrosis has been highlighted in previous studies, which were in agreement with our results $[11,19,20]$. Whereas, all of these studies were simply based on pathological observation. The role of collagen architectural features during regression of liver fibrosis was firstly verified by this objective data. The role of septum may be the key position in the process of collagen degradation.

Furthermore, septal width may be used to determine the reversibility of fibrosis. In previous studies, images analysis with traditional method did not allow for adequately refined quantification of septal histological features. Sethasine et al. [21] sought to quantify septal thickness and nodular size, but the irregular outline of the septa posed a challenge. The authors acknowledged that their method is cumbersome and not accurate enough. In our study, quantification of septal area was precisely segmented by image processing technology. We achieved precise identification of septal area and quantified septal width continually and automatically. Multivariate analysis of all related parameters indicated the importance of septal width. Also, a threshold of $30 \mu \mathrm{m}$ can be used to identify progressive vs. regressive septa.

One of the strengths of our study is the quantitative method with a stain-free SHG/TPEF-based microscopy technology. In previous studies, morphometric quantification to refine patterns of fibrotic changes during anti-viral treatment were based on collagen specific color-metric staining [22-27]. One commonly encountered difficulty was variation in staining, caused by the use of various batches in staining, time-dependent fading, and photo-bleaching [28]. Whereas, the stain-free technique presented in this study does not require specific staining steps and is easily standardized [29]. Besides, SHG provided higher signal-tobackground contrast and resolution, which facilitated 
making specific detection of fibrillar collagen [15]. Furthermore, the segmentation and quantification of septal area are fully automated, which is more time-saving and objective than traditional morphologic methods. Based on these characteristics, SHG represents a valuable tool for using in multi-center clinical trials.

Several limitations of our study should be acknowledged. First, because the collagen architectural pattern differs among different types of chronic liver disease, the importance of septal area during the regression of liver fibrosis may be limited to viral hepatitis; the role of septal area in other diseases needs to be further explored. Second, a sample size of 117 represents a limited data set. Although the follow-up period used in this study was relatively short, our study is ongoing and a third biopsy will be performed to evaluate the long-term histological outcomes. Third, the patients in this study were relatively younger than those with fibrosis/cirrhosis in the real-world [30]. The relatively young age of patients in this study was mainly due to the inclusion criteria. But we analyzed the effect of age on the reversal rate of liver fibrosis and found no significant difference between younger and older groups (Supplementary Table 4). Finally, the relatively high cost of this method renders it less popular in routine clinical laboratories. Despite these limitations, this study objectively demonstrates that septal area is the most predictive histological feature to evaluate the dynamic changes in liver fibrosis in chronic hepatitis B patients. Septal width was the most consistent indicator of prognosis in patients with fibrosis.

Acknowledgements This study was funded by National Science and Technology Major Project (2018ZX10302204), Key Project from Beijing Municipal Science and Technology Commission (D161100002716003, Z171100002217082), and National Natural Science Foundation of China (81670539). We thank Yayun Ren, who performed morphometric analyses, and Xiao Teng, who assisted with computer programming.

\section{Compliance with ethical standards}

Conflict of interest The authors declare that they have no conflict of interest.

Open Access This article is licensed under a Creative Commons Attribution 4.0 International License, which permits use, sharing, adaptation, distribution and reproduction in any medium or format, as long as you give appropriate credit to the original author(s) and the source, provide a link to the Creative Commons license, and indicate if changes were made. The images or other third party material in this article are included in the article's Creative Commons license, unless indicated otherwise in a credit line to the material. If material is not included in the article's Creative Commons license and your intended use is not permitted by statutory regulation or exceeds the permitted use, you will need to obtain permission directly from the copyright holder. To view a copy of this license, visit http://creativecommons. org/licenses/by/4.0/.

\section{References}

1. Schuppan D, Kim YO. Evolving therapies for liver fibrosis. J Clin Invest. 2013;123:1887-901.

2. Dienstag JL, Goldin RD, Heathcote EJ, et al. Histological outcome during long-term lamivudine therapy. Gastroenterology. 2003;124:105-17.

3. Hadziyannis SJ, Tassopoulos NC, Heathcote EJ, et al. Long-term therapy with adefovir dipivoxil for $\mathrm{HBeAg}$-negative chronic hepatitis B for up to 5 years. Gastroenterology. 2006;131:1743-51.

4. Chang TT, Liaw YF, Wu SS, et al. Long-term entecavir therapy results in the reversal of fibrosis/cirrhosis and continued histological improvement in patients with chronic hepatitis B. Hepatology. 2010;52:886-93.

5. Lok AS. Hepatitis: Long-term therapy of chronic hepatitis B reverses cirrhosis. Nat Rev Gastroenterol Hepatol. 2013; 10:199-200.

6. Marcellin P, Gane E, Buti M, et al. Regression of cirrhosis during treatment with tenofovir disoproxil fumarate for chronic hepatitis B: a 5-year open-label follow-up study. Lancet. 2013; 381:468-75.

7. Friedman SL. Mechanisms of hepatic fibrogenesis. Gastroenterology. 2008;134:1655-69.

8. Bedossa P. Reversibility of hepatitis B virus cirrhosis after therapy: who and why? Liver Int. 2015;35(Suppl 1):78-81.

9. Ishak K, Baptista A, Bianchi L, et al. Histological grading and staging of chronic hepatitis. J Hepatol. 1995;22:696-9.

10. Bedossa P, Poynard T. An algorithm for the grading of activity in chronic hepatitis C. The METAVIR Cooperative Study Group. Hepatology. 1996;24:289-93.

11. Wanless IR, Nakashima E, Sherman M. Regression of human cirrhosis. Morphologic features and the genesis of incomplete septal cirrhosis. Arch Pathol Lab Med. 2000;124:1599-607.

12. Cox G, Kable E, Jones A, et al. 3-dimensional imaging of collagen using second harmonic generation. J Struct Biol. 2003;141:53-62.

13. Sun W, Chang S, Tai DC, et al. Nonlinear optical microscopy: use of second harmonic generation and two-photon microscopy for automated quantitative liver fibrosis studies. J Biomed Opt. 2008; 13:064010

14. Tai DC, Tan N, Xu S, et al. Fibro-C-Index: comprehensive, morphology-based quantification of liver fibrosis using second harmonic generation and two-photon microscopy. J Biomed Opt. 2009;14:044013.

15. Gailhouste L, Le Grand Y, Odin C, et al. Fibrillar collagen scoring by second harmonic microscopy: a new tool in the assessment of liver fibrosis. J Hepatol. 2010;52:398-406.

16. Chen X, Nadiarynkh O, Plotnikov S, et al. Second harmonic generation microscopy for quantitative analysis of collagen fibrillar structure. Nat Protoc. 2012;7:654-69.

17. Xu S, Wang Y, Tai DC, et al. qFibrosis: a fully-quantitative innovative method incorporating histological features to facilitate accurate fibrosis scoring in animal model and chronic hepatitis B patients. J Hepatol. 2014;61:260-9.

18. Sun Y, Zhou J, Wang L, et al. New classification of liver biopsy assessment for fibrosis in chronic hepatitis B patients before and after treatment. Hepatology. 2017;65:1438-50.

19. Sandrini J, Boursier J, Chaigneau J, et al. Quantification of portalbridging fibrosis area more accurately reflects fibrosis stage and liver stiffness than whole fibrosis or perisinusoidal fibrosis areas in chronic hepatitis C. Mod Pathol. 2014;27:1035-45.

20. Chevallier M, Guerret S, Chossegros P, et al. A histological semiquantitative scoring system for evaluation of hepatic fibrosis in needle liver biopsy specimens: comparison with morphometric studies. Hepatology. 1994;20:349-55. 
21. Sethasine S, Jain D, Groszmann RJ, et al. Quantitative histological-hemodynamic correlations in cirrhosis. Hepatology. 2012;55:1146-53.

22. Kage M, Shimamatu K, Nakashima E, et al. Long-term evolution of fibrosis from chronic hepatitis to cirrhosis in patients with hepatitis C: morphometric analysis of repeated biopsies. Hepatology. 1997;25:1028-31.

23. Duchatelle V, Marcellin P, Giostra E, et al. Changes in liver fibrosis at the end of alpha interferon therapy and 6 to 18 months later in patients with chronic hepatitis C: quantitative assessment by a morphometric method. J Hepatol. 1998;29:20-8.

24. Caballero T, Perez-Milena A, Masseroli M, et al. Liver fibrosis assessment with semiquantitative indexes and image analysis quantification in sustained-responder and non-responder interferon-treated patients with chronic hepatitis C. J Hepatol. 2001;34:740-7.

25. Arima $\mathrm{M}$, Terao $\mathrm{H}$, Kashima $\mathrm{K}$, et al. Regression of liver fibrosis in cases of chronic liver disease type C: quantitative evaluation by using computed image analysis. Intern Med. 2004;43:902-10.

26. Goodman ZD, Stoddard AM, Bonkovsky HL, et al. Fibrosis progression in chronic hepatitis $\mathrm{C}$ : morphometric image analysis in the HALT-C trial. Hepatology. 2009;50:1738-49.

27. Huang Y, de Boer WB, Adams LA, et al. Image analysis of liver biopsy samples measures fibrosis and predicts clinical outcome. J Hepatol. 2014;61:22-7.

28. Cales P, Chaigneau J, Hunault G, et al. Automated morphometry provides accurate and reproducible virtual staging of liver fibrosis in chronic hepatitis C. J Pathol Inform. 2015;6:20.

29. Zhuang ZF, Zhu MF, Guo ZY, et al. Second harmonic generation in 3-d uniform arrangement of type I collagen on nonlinear optics microscopy. Scanning. 2013;35:12-6.

30. You H, Kong Y, Hou J, et al. Female gender lost protective effect against disease progression in elderly patients with chronic hepatitis B. Sci Rep. 2016;6:37498. 